Part of Journal of Research of the National Bureau of Standards, Volume 20, January 1938

\title{
INFRARED SPECTRA OF IRON, TITANIUM, AND CARBON
}

\author{
By Carl C. Kiess
}

\section{ABSTRACT}

New wave lengths in the arc spectra of iron and titanium have been recorded photographically with the type $Z$ plates produced by the Kodak Research Laboratories. The long wave-length limits of these spectra have now been extended to the vicinity of $12000 \mathrm{~A}$. Many of the observed lines were predictable from previously known terms. Several apparently related groups of lines in titanium have revealed a new term, $e^{\prime 3} \mathrm{P}$, belonging to the third set and combining with terms of the middle set. Previously known lines in the spectrum of carbon have been photographed with high dispersion to obtain accurate wave-length values for use in identifying infrared lines in the sun's spectrum. A new band in the infrared system of $\mathrm{CN}$ has been found.

\section{CONTENTS}

I. Introduction

II. Results

1. Iron

3. Carbon.

\section{INTRODUCTION}

In this journal, ${ }^{1}$ five years ago, were presented descriptions of the infrared spectra of several elements which, with the aid of the new types of plates brought out by the Research Laboratories of the Eastman Kodak Co., extended the limits of photographically recorded wave lengths to the neighborhood of $11000 \mathrm{~A}$. Since that time new sensitizers, the pentacarbocyanines, ${ }^{2}$ have been synthesized, with which plates, sensitive out to wave length $13000 \mathrm{~A}$ and beyond, have been prepared. It is the purpose of this paper to present the results that have been obtained with these new plates for iron, titanium, and carbon. The methods and apparatus employed in this investigation were the same as those described in the earlier work mentioned above.

\section{RESULTS}

\section{IRON}

The new infrared wave lengths of iron are listed in table 1 . These lines were recorded on the $Z$ plates with exposures, ranging from 1 to 2 hours, to a d-c arc operated on 6 or 8 amperes from a 220 -volt circuit. The intensities given for the lines are the usual eye estimates, but from the low order of the assigned numbers it can be seen that

1 W. F. Meggers and C. C. Kiess, BS J. Research, 9, 309(1932) RP473.

2 L. S. G. Brooker and G. H. Keyes, J. Franklin Inst. 219, 255(1935). 
in this region iron has no lines of suitable strength for standards. The classifications of the lines given in the last column are based on known terms taken from the work of Burns and Walters, ${ }^{3}$ or from Catalán. ${ }^{4}$ No new terms have been found.

TABLE 1.-Wave lengths in the infrared spectrum of iron

\begin{tabular}{|c|c|c|c|}
\hline$\lambda_{\text {gir }} \mathrm{A}$ & $\begin{array}{l}\text { Inten- } \\
\text { sity }\end{array}$ & $\nu_{\mathrm{vaO}} \mathrm{cm}^{-1}$ & Term combination \\
\hline $\begin{array}{l}\text { 11973. } 01 \\
11884.12 \\
11882.80 \\
11783.28 \\
11689.98\end{array}$ & $\begin{array}{l}8 \\
3 \\
7 \\
6 \\
8\end{array}$ & $\begin{array}{l}\text { 8349. } 84 \\
8412.29 \\
8413.22 \\
8484.28 \\
8551.99\end{array}$ & $\begin{array}{l}a^{5} \mathrm{P}_{3}-z^{5} \mathrm{D}_{4}^{\circ} \\
a^{5} \mathrm{P}_{1}-z^{5} \mathrm{D}_{2}^{\circ} \\
a^{5} \mathrm{P}_{2}-z^{5} \mathrm{D}_{3}^{3} \\
b^{3} \mathrm{P}_{2}-z^{3} \mathrm{D}_{3}^{\circ} \\
a^{5} \mathrm{P}_{1}-z^{5} \mathrm{D}_{1}^{\circ}\end{array}$ \\
\hline $\begin{array}{l}11638.25 \\
11607.57 \\
11593.55 \\
11439.06 \\
11422.30\end{array}$ & $\begin{array}{r}7 \\
12 \\
5 \\
15 \\
6\end{array}$ & $\begin{array}{l}8590.00 \\
8612.70 \\
8623.13 \\
8739.59 \\
8752.41\end{array}$ & $\begin{array}{l}a^{5} \mathrm{P}_{3}-z^{5} \mathrm{D}_{3}^{\circ} \\
a^{5} \mathrm{P}_{2}-z^{5} \mathrm{D}_{2}^{\circ} \\
a^{5} \mathrm{P}_{1}-z^{5} \mathrm{D}_{0}^{\circ} \\
b^{3} \mathrm{P}_{1}-z^{3} \mathrm{D}_{2}^{\circ} \\
a^{5} \mathrm{P}_{2}-z^{5} \mathrm{D}_{1}^{\circ}\end{array}$ \\
\hline $\begin{array}{l}11374.02 \\
11355.97 \\
11298.83 \\
11251.09 \\
11149.34\end{array}$ & $\begin{array}{l}3 \\
1 \\
3 \\
3 \\
2\end{array}$ & $\begin{array}{l}8789.56 \\
8803.53 \\
8848.05 \\
8885.60 \\
8966.69\end{array}$ & $\begin{array}{l}a^{5} \mathrm{P}_{3}-z^{5} \mathrm{D}_{2}^{\circ} \\
b^{3} \mathrm{P}_{2}-z^{3} \mathrm{D}_{2}^{\circ} \\
b^{3} \mathrm{P}_{0}-z^{3} \mathrm{D}_{1}^{\circ} \\
b^{3} \mathrm{P}_{2}-z^{3} \mathrm{~F}_{2}^{\circ}\end{array}$ \\
\hline $\begin{array}{l}11119.80 \\
11045.6 \\
11013.27 \\
11004.28 \\
10991.70\end{array}$ & $\begin{array}{l}10 \\
1 h \\
1 \\
1 \\
1\end{array}$ & $\begin{array}{l}8990.51 \\
9050.92 \\
9077.47 \\
9084.89 \\
9095.28\end{array}$ & $\begin{array}{l}b^{3} \mathrm{P}_{1}-z^{3} \mathrm{D}_{1}^{\circ} \\
y^{3} \mathrm{D}_{2}^{\circ}-e^{5} \mathrm{~F}_{3}\end{array}$ \\
\hline $\begin{array}{l}10946.52 \\
10936.00 \\
10925.80 \\
10896.30 \\
10886.81\end{array}$ & $\begin{array}{l}1 \\
1 \\
1 \\
3 \\
1\end{array}$ & $\begin{array}{l}9132.82 \\
9141.61 \\
9150.14 \\
9174.92 \\
9182.91\end{array}$ & $4 \mathrm{M}_{2}-z^{3} \mathrm{P}_{2}^{\circ}$ \\
\hline $\begin{array}{l}10884.30 \\
10881.65 \\
10863.60 \\
10849.68 \\
10845.62\end{array}$ & $\begin{array}{l}3 \\
1 \\
5 \\
2 \\
1\end{array}$ & $\begin{array}{l}9185.03 \\
9187.27 \\
9202.53 \\
9214.34 \\
9217.79\end{array}$ & $\begin{array}{l}b^{3} \mathrm{P}_{1}-z^{3} \mathrm{~F}_{2}^{\circ} \\
y^{3} \mathrm{D}_{3}^{\circ}-e^{5} \mathrm{~F}_{4}\end{array}$ \\
\hline $\begin{array}{l}\text { 10825. } 32 \\
10818.36 \\
10783.09 \\
10760.62 \\
10752.99\end{array}$ & $\begin{array}{l}1 \\
3 \\
3 \\
1 \\
3\end{array}$ & $\begin{array}{l}9235.08 \\
9241.02 \\
9271.24 \\
9290.61 \\
9297.20\end{array}$ & \\
\hline $\begin{array}{l}10735.46 \\
10718.38 \\
10532.21 \\
10469.59 \\
10452.70\end{array}$ & $\begin{array}{r}2 \\
2 \\
10 \\
20 \\
5\end{array}$ & $\begin{array}{l}9312.37 \\
9327.22 \\
9492.09 \\
9548.85 \\
9564.29\end{array}$ & \\
\hline $\begin{array}{l}10435.17 \\
10423.65 \\
10422.99 \\
10400.94\end{array}$ & $\begin{array}{l}0 b \\
3 \\
0 \\
1\end{array}$ & $\begin{array}{l}9580.36 \\
9590.95 \\
9591.55 \\
9611.88\end{array}$ & $\begin{array}{l}y^{3} \mathrm{D}_{3}^{\circ}-e^{5} \mathrm{~F}_{3} \\
4 \mathrm{M}_{2}-z^{3} \mathrm{P}_{1}^{\circ} \\
a^{3} \mathrm{G}_{5}-z^{3} \mathrm{~F}_{4}^{\circ}\end{array}$ \\
\hline
\end{tabular}

${ }^{3}$ K. Burns and F. M. Walters, jr. Pub. Allegheny Observatory 6, 159(1929).

- M. A. Catalán. Anales soc. españ. fís. quím. 28, 1239(1930). 


\section{TITANIUM}

Nearly all the new lines of $\mathrm{Ti}$ I listed in table 2 were predictable from the previously known terms of this spectrum. These terms, owing largely to the work of Russell, ${ }^{5}$ are listed by Miss Moore with the designations or symbols now in use. ${ }^{6}$ To these is added a new term, $e^{\prime 3} \mathrm{P}$, revealed by the present investigation. Its components, with their inner quantum numbers, are:

$$
\begin{array}{cr}
e^{\prime}{ }^{3} \mathrm{P}_{2} & 34535.04 \\
& 207.08 \\
e^{\prime}{ }^{3} \mathrm{P}_{1} & 34327.96 \\
& 157.01 \\
e^{\prime}{ }^{3} \mathrm{P}_{0} & 34170.95
\end{array}
$$

\begin{tabular}{|c|c|c|c|}
\hline$\lambda_{\text {air }} \mathrm{A}$ & Intensity & $\nu_{\mathrm{v} a \mathrm{e}} \mathrm{cm}^{-1}$ & Term combination \\
\hline $\begin{array}{l}11973.88 \\
11949.58 \\
11892.85\end{array}$ & $\begin{array}{l}6 \\
5 \\
5\end{array}$ & $\begin{array}{l}\text { 8349. } 23 \\
8366.20 \\
8406.12\end{array}$ & $\begin{array}{l}b^{3} \mathrm{~F}_{4}-z^{3} \mathrm{D}_{3}^{\circ} \\
b^{3} \mathrm{~F}_{3}-z^{3} \mathrm{D}_{2}^{\circ} \\
b^{3} \mathrm{~F}_{2}-z^{3} \mathrm{D}_{1}^{\circ}\end{array}$ \\
\hline 11830. 36 & $0 b$ & 8450.51 & $\begin{array}{l}v^{3} \mathrm{D}_{2}^{\circ}-f^{3} \mathrm{~F}_{3} \\
v^{3} \mathrm{G}^{\circ}-e^{3} \mathrm{G}_{5}\end{array}$ \\
\hline 11797. 24 & 3 & 8474. 24 & $b^{3} \mathrm{~F}_{2}-z^{3} \mathrm{D}_{2}^{\circ}$ \\
\hline $\begin{array}{l}11780.54 \\
11675.11\end{array}$ & $\begin{array}{l}4 \\
2\end{array}$ & $\begin{array}{l}8486.25 \\
8562.88\end{array}$ & $b^{3} \mathrm{~F}_{3}-z^{3} \mathrm{D}_{3}^{\circ}$ \\
\hline 11667.46 & 2 & 8568. 50 & $c^{3} \mathrm{P}_{2}-x^{3} \mathrm{D}_{3}^{\circ}$ \\
\hline $\begin{array}{l}11652.16 \\
11647.48\end{array}$ & $\begin{array}{l}0 \\
0\end{array}$ & $\begin{array}{l}8579.75 \\
8583.20\end{array}$ & $v^{3} \mathrm{D}_{3}^{\circ}-f^{3} \mathrm{~F}_{4}$ \\
\hline $\begin{array}{l}11641.91 \\
11635.16 \\
11609.41 \\
11539.50 \\
11403.89\end{array}$ & $\begin{array}{l}0 \\
0 \\
3 \\
5 \\
8\end{array}$ & $\begin{array}{l}\text { 8587. } 30 \\
8592.29 \\
8611.34 \\
8663.51 \\
8766.54\end{array}$ & $c^{3} \mathrm{P}_{1}-x^{3} \mathrm{D}_{2}^{\circ}$ \\
\hline $\begin{array}{l}11381.53 \\
11354.90 \\
11316.72 \\
11292.43 \\
11246.88\end{array}$ & $\begin{array}{l}7 \\
2 \\
2 \\
6 \\
8\end{array}$ & $\begin{array}{l}8783.76 \\
8804.36 \\
8834.06 \\
8853.07 \\
8888.92\end{array}$ & $\begin{array}{l}{ }^{2}{ }^{3} \mathrm{P}_{2}^{\circ}-e^{\prime}{ }^{3} \mathrm{P}_{1} \\
y^{3} \mathrm{D}_{1}^{\circ}-e^{\prime}{ }^{3} \mathrm{P}_{0} \\
y^{3} \mathrm{D}_{2}^{\circ}-e^{\prime}{ }^{3} \mathrm{P}_{1}\end{array}$ \\
\hline $\begin{array}{l}11243.90 \\
11230.91 \\
11144.74 \\
11095.79 \\
11057.58\end{array}$ & $\begin{array}{r}10 \\
5 \\
1 \\
5 \\
3\end{array}$ & $\begin{array}{l}8891.28 \\
8901.56 \\
8970.39 \\
9009.96 \\
9041.08\end{array}$ & $\begin{array}{l}y^{3} \mathrm{D}_{3}^{\circ}-e^{\prime}{ }^{3} \mathrm{P}_{2} \\
y^{3} \mathrm{D}_{1}^{\circ}-e^{\prime}{ }^{3} \mathrm{P}_{1} \\
z^{3} \mathrm{P}_{2}^{\circ}-e^{\prime}{ }^{3} \mathrm{P}_{2}\end{array}$ \\
\hline $\begin{array}{l}\text { 10990. } 70 \\
\text { 10977. } 90 \\
10974.50 \\
10905.08 \\
10899.13\end{array}$ & $\begin{array}{l}3 \\
2 \\
2 \\
1 \\
1\end{array}$ & $\begin{array}{l}9096.11 \\
9106.72 \\
9109.54 \\
9167.53 \\
9172.54\end{array}$ & $\begin{array}{l}y^{3} \mathrm{D}_{2}^{\circ}-e^{\prime}{ }^{3} \mathrm{P}_{2} \\
x^{3} \mathrm{~F}_{4}^{\circ}-e^{3} \mathrm{G}_{4} \\
x^{3} \mathrm{~F}_{3}^{\circ}-e^{3} \mathrm{G}_{3}\end{array}$ \\
\hline $\begin{array}{l}10896.10 \\
10855.15 \\
10853.14 \\
10847.72 \\
10833.66\end{array}$ & $\begin{array}{l}8 \\
1 \\
3 \\
1 \\
3\end{array}$ & $\begin{array}{l}\text { 9174. } 24 \\
9209.70 \\
9211.40 \\
9216.00 \\
9227.97\end{array}$ & $\begin{array}{l}a^{5} \mathrm{~F}_{3}-z^{5} \mathrm{G}_{2}^{\circ} \\
x^{5} \mathrm{D}_{3}^{\circ}-f^{5} \mathrm{~F}_{3}\end{array}$ \\
\hline
\end{tabular}

TABLE 2.-Wave lengths in the infrared spectrum of titanium

${ }^{\circ}$ H. N. Russell. Astrophys. J. 66, 347 (1927).

C. E. Moore. Term Designations for Excitation Potentials, p. 12, (Princeton University Observatory, 1934). 
TABLE 2.-Wave lengths in the infrared spectrum of titaniumContinued

\begin{tabular}{|c|c|c|c|}
\hline$\lambda_{\mathrm{air}} \mathrm{A}$ & Intensity & $\nu_{\mathrm{vac}} \mathrm{cm}^{-1}$ & Term combination \\
\hline $\begin{array}{l}10828.04 \\
10820.31\end{array}$ & $\frac{1}{5}$ & $\begin{array}{l}9232.76 \\
9239.35\end{array}$ & $\begin{array}{l}a^{5} \mathrm{~F}_{4}-z^{5} \mathrm{G}_{3} \\
x^{3} \mathrm{~F}_{3}^{\circ}-e^{3} \mathrm{G}_{4}\end{array}$ \\
\hline 10817. 35 & $5 b$ & 9241.88 & $x^{5} \mathrm{D}_{2}^{\circ}-f^{5} \mathrm{~F}_{2}$ \\
\hline $\begin{array}{l}10806.03 \\
10793.65\end{array}$ & $\begin{array}{l}2 \\
3\end{array}$ & $\begin{array}{l}9251.56 \\
9262.18\end{array}$ & $\begin{array}{l}x^{5} \mathrm{D}_{1}^{\circ}-f^{5} \mathrm{~F}_{1} \\
x^{3} \mathrm{~F}_{2}^{\circ}-e^{3} \mathrm{G}_{3}\end{array}$ \\
\hline 10781. 34 & 3 & 9272.75 & $b^{3} \mathrm{P}_{2}-x^{3} \mathrm{D}_{2}^{\circ}$ \\
\hline 10774.92 & 12 & 9278.27 & $\begin{array}{l}x^{5} \mathrm{D}_{0}^{0}-f^{0} \mathrm{~F}_{1} \\
a^{5} \mathrm{~F}_{2}-z^{5} \mathrm{G}_{2}^{\circ}\end{array}$ \\
\hline 10756.90 & 5 & 9293. 82 & $b^{3} \mathrm{P}_{1}-x^{3} \mathrm{D}_{1}^{\circ}$ \\
\hline $\begin{array}{l}10751.80 \\
10747.62\end{array}$ & $\begin{array}{l}1 \\
1 b\end{array}$ & $\begin{array}{l}9298.23 \\
9301.84\end{array}$ & \\
\hline $\begin{array}{l}10741.77 \\
10732.89 \\
10731.11 \\
10726.33 \\
10690.88\end{array}$ & $\begin{array}{r}7 \\
8 \\
6 \\
18 \\
1\end{array}$ & $\begin{array}{l}9306.91 \\
9314.60 \\
9316.15 \\
9320.30 \\
9351.20\end{array}$ & $\begin{array}{l}x^{5} \mathrm{D}_{2}^{\circ}-f^{5} \mathrm{~F}_{3} \\
a^{5} \mathrm{~F}_{3}-z^{5} \mathrm{G}_{3}^{\circ} \\
x^{5} \mathrm{D}_{3}^{\circ}-f^{5} \mathrm{~F}_{4} \\
a^{5} \mathrm{~F}_{1}-z^{5} \mathrm{G}_{2}^{\circ}\end{array}$ \\
\hline $\begin{array}{l}10689.52 \\
10677.04 \\
10661.61 \\
10646.68 \\
10627.67\end{array}$ & $\begin{array}{r}15 \\
10 \\
20 \\
3 \\
1\end{array}$ & $\begin{array}{l}9352.39 \\
9363.33 \\
9376.88 \\
9390.91 \\
9406.82\end{array}$ & $\begin{array}{c}x^{5} \mathrm{D}_{4}^{\circ}-f^{5} \mathrm{~F}_{5} \\
a{ }^{5} \mathrm{~F}_{4}-z^{5} \mathrm{G}_{4} \\
a^{5} \mathrm{~F}_{2}-z^{5} \mathrm{G}_{3}^{\circ} \\
z^{3} \mathrm{~S}_{1}-e^{\prime}{ }^{3} \mathrm{P}_{1}\end{array}$ \\
\hline $\begin{array}{l}10607.78 \\
10594.90 \\
10591.69 \\
10589.75 \\
10584.66\end{array}$ & $\begin{array}{r}10 \\
2 \\
1 \\
2 \\
25\end{array}$ & $\begin{array}{l}9424.46 \\
9435.92 \\
9438.78 \\
9440.51 \\
9445.05\end{array}$ & $\begin{array}{c}a^{5} \mathrm{~F}_{5}-z^{5} \mathrm{G}_{5} \\
y^{5} \mathrm{G}_{5}^{\circ}-e^{5} \mathrm{~F}_{4} \\
y^{5} \mathrm{G}_{4}^{\circ}-e^{5} \mathrm{~F}_{3} \\
y^{5} \mathrm{G}_{6}^{\circ}-e^{5} \mathrm{~F}_{5} \\
a^{5} \mathrm{~F}_{3}-z^{5} \mathrm{G}_{4}^{\circ}\end{array}$ \\
\hline $\begin{array}{l}10565.97 \\
10553.02 \\
10551.81 \\
10548.64 \\
10496.14\end{array}$ & $\begin{array}{c}5 \\
8 \\
3 \\
2 b \\
30\end{array}$ & $\begin{array}{l}9461.76 \\
9473.37 \\
9474.45 \\
9477.30 \\
9524.71\end{array}$ & $\begin{array}{l}a^{3} \mathrm{H}_{4}-y^{3} \mathrm{G}_{3} \\
a^{3} \mathrm{H}_{5}-y^{3} \mathrm{G}_{4}^{\circ} \\
a^{3} \mathrm{G}_{5}-z^{1} \mathrm{G}_{4}^{\circ} \\
a^{5} \mathrm{~F}_{4}-z^{5} \mathrm{G}_{5}^{\circ}\end{array}$ \\
\hline $\begin{array}{l}10460.07 \\
10453.42 \\
10415.53 \\
10400.84 \\
10398.93\end{array}$ & $\begin{array}{l}10 \\
2 b \\
2 b \\
1 \\
1\end{array}$ & $\begin{array}{l}9557.55 \\
9563.63 \\
9598.42 \\
9611.97 \\
9613.74\end{array}$ & $\begin{array}{l}a^{3} \mathrm{H}_{6}-y^{3} \mathrm{G}_{5}^{\circ} \\
x^{3} \mathrm{G}_{3}-f^{3} \mathrm{~F}_{2} \\
z^{3} \mathrm{~S}_{1}^{\circ}-e^{\prime}{ }^{3} \mathrm{P}_{2}\end{array}$ \\
\hline $\begin{array}{l}10396.85 \\
10338.59 \\
10257.30 \\
10189.26 \\
10179.92\end{array}$ & $\begin{array}{l}25 \\
2 b \\
3 b \\
3 \\
3 b\end{array}$ & $\begin{array}{l}9615.66 \\
9669.85 \\
9746.48 \\
9811.57 \\
9820.48\end{array}$ & $\begin{array}{c}a^{5} \mathrm{~F}_{5}-z^{5} \mathrm{G}_{6} \\
x^{3} \mathrm{G}_{4}^{\circ}-f^{3} \mathrm{~F}_{3} \\
x^{3} \mathrm{G}_{5}^{\circ}-f^{3} \mathrm{~F}_{4} \\
b^{3} \mathrm{~F}_{4}-z^{3} \mathrm{G}_{4}^{\circ} \\
w^{3} \mathrm{G}_{3}^{\circ}-f^{3} \mathrm{G}_{3}\end{array}$ \\
\hline $\begin{array}{l}10170.60 \\
10147.09 \\
10145.48 \\
10120.90 \\
10119.20\end{array}$ & $\begin{array}{r}3 \\
4 \\
8 \\
10 \\
3\end{array}$ & $\begin{array}{l}9829.57 \\
9852.34 \\
9853.91 \\
9877.84 \\
9879.50\end{array}$ & $\begin{array}{c}b^{3} \mathrm{~F}_{3}-z^{3} \mathrm{G}_{3}^{\circ} \\
w^{3} \mathrm{G}_{5}^{\circ}-f^{3} \mathrm{G}_{5} \\
a^{3} \mathrm{D}_{3}-x^{3} \mathrm{D}_{2}^{\circ} \\
w^{3} \mathrm{G}_{4}^{\circ}-f^{3} \mathrm{G}_{4}\end{array}$ \\
\hline
\end{tabular}




\section{CARBON}

The lines of the neutral carbon atom given in table 3 were measured to obtain improved wave lengths for use in identification of infrared solar lines. These carbon lines were previously known, partly from the work of Ryde, ${ }^{7}$ but mainly from that of Ingram, ${ }^{8}$ who gave the classifications. However, the observations of these investigators yielded only approximate values for the wave lengths. Edlén ${ }^{9}$ has recently given more accurate measurements. In the present work, the wave lengths are derived from spectrograms taken in the first order of the large concave grating ruled with 20,000 lines per inch and having a dispersion of $3.6 \mathrm{~A} / \mathrm{mm}$. The light source was a condensed discharge through a Geissler tube containing $\mathrm{CO}_{2}$ at a pressure of approximately $5 \mathrm{~mm}$. These lines are also observable in the spectrum of an arc between graphite electrodes, but they are broadened in the arc and are affected by blending with lines belonging to the bands with heads at 9140 and $10925 \mathrm{~A}$. The former of these bands was first recorded photographically by Asundi and Ryde,,$^{10}$ who tentatively designated it as the $(0,0)$ band of the ${ }^{2} \Pi \rightarrow \rightarrow^{2} \Sigma$ system of $\mathrm{CN}$. Subsequently, it was photographed with higher dispersion by Parker, ${ }^{11}$ who has given an analysis of it. The band at $10925 \mathrm{~A}$ is new and is apparently a member of the same system as the one at $9140 \mathrm{~A}$. It is traceable on our plates out to $11800 \mathrm{~A}$ and is accompanied by a much fainter band with head at $10872 \mathrm{~A}$.

TABLE 3.-Wave lengths in the infrared spectrum of carbon

\begin{tabular}{|c|c|c|c|}
\hline$\lambda_{\text {air }} \mathrm{A}$ & Intensity & $\nu_{\mathrm{va} a} \mathrm{~cm}^{-1}$ & Term combination \\
\hline $\begin{array}{l}11330.36 \\
10729.59 \\
10707.44 \\
10691.36 \\
10685.44\end{array}$ & $\begin{array}{r}1 \\
8 \\
8 \\
50 \\
10\end{array}$ & $\begin{array}{l}8823.43 \\
9317.47 \\
9336.75 \\
9350.78 \\
9355.97\end{array}$ & $\begin{array}{l}3 p^{1} \mathrm{P}_{1}-3 d^{1} \mathrm{D}_{2}^{\circ} \\
3 s^{3} \mathrm{P}_{2}^{\circ}-3 p^{3} \mathrm{D}_{2} \\
3 s^{3} \mathrm{P}_{1}^{\circ}-3 p^{3} \mathrm{D}_{1} \\
3 s^{3} \mathrm{P}_{2}^{\circ}-3 p^{3} \mathrm{D}_{3} \\
3 s^{3} \mathrm{P}_{0}^{\circ}-3 p^{3} \mathrm{D}_{1}\end{array}$ \\
\hline $\begin{array}{r}10683.18 \\
9658.49 \\
9620.86 \\
9603.09 \\
9405.77\end{array}$ & $\begin{array}{r}25 \\
2 \\
1 \\
0 \\
20\end{array}$ & $\begin{array}{r}9357.95 \\
10350.75 \\
10391.23 \\
10410.46 \\
10628.86\end{array}$ & $\begin{array}{l}3 s^{3} \mathrm{P}_{1}^{\circ}-3 p^{3} \mathrm{D}_{2} \\
3 s^{3} \mathrm{P}_{2}^{\circ}-3 p^{3} \mathrm{~S}_{1} \\
3 s^{3} \mathrm{P}_{1}^{\circ}-3 p^{3} \mathrm{~S}_{1} \\
3 s^{3} \mathrm{P}_{0}^{\circ}-3 p^{3} \mathrm{~S}_{1} \\
3 s^{1} \mathrm{P}_{1}^{\circ}-3 p^{1} \mathrm{D}_{2}\end{array}$ \\
\hline $\begin{array}{l}9111.85 \\
9094.89 \\
9088.57 \\
9078.32 \\
9062.53\end{array}$ & $\begin{array}{r}10 \\
25 \\
8 \\
6 \\
10\end{array}$ & $\begin{array}{l}\text { 10971. } 71 \\
10992.17 \\
10999.81 \\
11012.23 \\
11031.42\end{array}$ & $\begin{array}{l}3 s^{3} \mathrm{P}_{2}^{\circ}-3 p^{3} \mathrm{P}_{1} \\
3 s^{3} \mathrm{P}_{2}^{\circ}-3 p^{3} \mathrm{P}_{2} \\
3 s^{3} \mathrm{P}_{1}^{\circ}-3 p^{3} \mathrm{P}_{0} \\
3 s^{3} \mathrm{P}_{1}^{\circ}-3 p^{3} \mathrm{P}_{1} \\
3 s^{3} \mathrm{P}_{0}^{\circ}-3 p^{3} \mathrm{P}_{1}\end{array}$ \\
\hline $\begin{array}{l}9061.48 \\
8335.19\end{array}$ & $\begin{array}{l}15 \\
10\end{array}$ & $\begin{array}{l}\text { 11032. } 70 \\
11994.03\end{array}$ & $\begin{array}{l}3 s^{3} \mathrm{P}_{\mathrm{i}}-3 p{ }^{3} \mathrm{P}_{2} \\
3 s^{1} \mathrm{P}_{1}^{\circ}-3 p{ }^{1} \mathrm{~S}_{0}\end{array}$ \\
\hline
\end{tabular}

7 J. W. Ryde, Proc. Roy. Soc. (London) A117, 164 (1927).

8 S. B. Ingram, Phys. Rev., 34, 421 (1929).

B. Edlén, Z. Physik 98, 459 (1936).

10 R. K. Asundi and J. W. Ryde, Nature 124, 57 (1929).

11 A. E. Parker, Phys. Rev. 41, 274 (1932).

Washington, November 17, 1937. 